ITP-SB-97-30

\title{
A Mapping Relating Complex and Physical Temperatures in the 2D $q$-State Potts Model and Some Applications
}

\author{
Heiko Feldmann‡, Robert Shrock ${ }^{\text {** }}$ and Shan-Ho Tsai \\ Institute for Theoretical Physics \\ State University of New York \\ Stony Brook, N. Y. 11794-3840
}

\begin{abstract}
We show an exact equivalence of the free energy of the $q$-state Potts antiferromagnet on a lattice $\Lambda$ for the full temperature interval $0 \leq T \leq \infty$ and the free energy of the $q$-state Potts model on the dual lattice for a semi-infinite interval of complex temperatures (CT). This implies the existence of two quite different types of CT singularities: the generic kind, which does not obey universality or various scaling relations, and a special kind which does obey such properties and encodes information of direct physical relevance. We apply this observation to characterize CT properties of the Potts model on several lattices, to rule out two existing conjectures, and to determine the critical value of $q$ above which the Potts antiferromagnet on the diced lattice has no phase transition.
\end{abstract}

*email: feldmann@insti.physics.sunysb.edu

**email: shrock@insti.physics.sunysb.edu

${ }^{\dagger \dagger}$ email: tsai@insti.physics.sunysb.edu 
The study of statistical mechanical models with magnetic field [1], temperature [2]-[团, or both [5] generalized from real to complex values has yielded interesting insights into the properties of these models. Complex-temperature (CT) singularities have been shown to have, in general, rather different properties than physical critical points of spin models, including violation of exponent relations such as $\gamma \neq \gamma^{\prime}$ [6] and violation of universality, as evidenced by lattice dependence of exponents (shown using exact results) [7]. It is therefore of fundamental interest to understand better how CT properties of spin models are related to physical properties. Here we shall show an exact equivalence of the free energy of the $q$-state Potts antiferromagnet on a lattice $\Lambda$ for the full temperature interval $0 \leq T \leq \infty$ and the free energy of the $q$-state Potts model $(q \mathrm{PM})$ on the dual lattice for the interval $-\infty \leq \exp \left(J /\left(k_{B} T\right)\right) \leq-(q-1)$, where $J$ denotes the spin-spin coupling. This result leads to the important conclusion that there are two different types of CT singularities: the generic type, with violations of scaling and universality, and a special type, which is closely related to physical critical points and can be described by the same ideas of scaling, renormalization group (RG), universality classes determined as RG fixed points and related, for 2D models, to conformal field theories. Our observation also yields a way to determine some properties of certain theories with non-Gibbs measures, by relating them exactly to theories with Gibbs measures. This is a useful connection, since theories with non-Gibbs measures appear not just in the context of complex temperature or magnetic field, but also in a number of physical situations, such as quantum spin models in condensed matter, lattice quantum chromodynamics with finite chemical potential or topological charge, and the lattice formulation of the standard $\mathrm{SU}(2) \times \mathrm{U}(1)$ electroweak gauge theory.

The $q$-state Potts model [8]-10] with zero external field(s) on a lattice $\Lambda$ is defined by the partition function $Z=\sum_{\left\{\sigma_{n}\right\}} \exp (-\beta \mathcal{H})$ with the Hamiltonian

$$
\mathcal{H}=-J \sum_{\left\langle n n^{\prime}\right\rangle} \delta_{\sigma_{n} \sigma_{n^{\prime}}}
$$

in standard notation, where $\sigma_{n}=1, \ldots, q, \beta=\left(k_{B} T\right)^{-1}$, and $\left\langle n n^{\prime}\right\rangle$ denotes pairs of nearestneighbor sites on $\Lambda$. We also define $K=\beta J$,

$$
a=z^{-1}=e^{K}, \quad x=\frac{e^{K}-1}{\sqrt{q}}
$$

We consider $d=2$ dimensional lattices, since in this case the model with spin-spin interactions along bonds maps to another with similar interactions on the bonds of the dual lattice. Physical applications of the 2D ferromagnetic (FM) Potts model for $q=3$ and 4 include modelling properties of monolayers of gas molecules absorbed on substrates. The 2D Potts 
ferromagnet is known to have a $\left(\mathbb{Z}_{q}\right.$ symmetry-breaking) phase transition which is secondorder for $2 \leq q \leq 4$ and first order for $q \geq 5$. The critical exponents and universality classes of the cases where the model has second-order transitions are well understood [9, 11], but, aside from the $q=2$ Ising case [12], the free energy has never been calculated for general $T$. The Potts antiferromagnet has also been of interest because of its connection with graph colorings and the fact that, for certain lattices and values of $q$, it exhibits nonzero ground state entropy.

Now consider the $q \mathrm{PM}$ on a $2 \mathrm{D}$ lattice $\Lambda$ with $N_{0}$ sites (0-cells), $N_{1}$ bonds (1-cells) and $N_{2}$ faces (2-cells). The dual lattice $\Lambda_{d}=\mathcal{D}(\Lambda)$ is defined by associating uniquely a $(2-p)$-cell of $\Lambda_{d}$ with each $p$-cell of $\Lambda$. The partition function of the $q \mathrm{PM}$ satisfies the duality relation 8, 9, 13.

$$
Z(\Lambda, q, a)=x^{N_{1}} q^{N_{0}-1-(1 / 2) N_{1}} Z\left(\Lambda_{d}, q, a_{d}\right)
$$

where

$$
a_{d} \equiv \mathcal{D}(a)=\frac{a+q-1}{a-1}, \quad \text { i.e. } \quad x_{d}=\frac{1}{x}
$$

It follows from (3) that in the thermodynamic limit, the singularities of the free energy of the $q \mathrm{PM}$ on $\Lambda$ at a point $a$ satisfy

$$
f(\Lambda, q, a)_{\text {sing }}=f\left(\Lambda_{d}, q, a_{d}\right)_{\text {sing }}
$$

The key observation is that under duality, the complete physical temperature interval $0 \leq$ $T \leq \infty$, i.e., $0 \leq a \leq 1$ of the $q$-state Potts antiferromagnet (qPAF) on $\Lambda$ is mapped to the CT interval $-\infty \leq a_{d} \leq-(q-1)$ on $\Lambda_{d}$ and vice versa. (In contrast, the physical $T$ interval of the $q$-state Potts ferromagnet on $\Lambda, 1 \leq a \leq \infty$, maps under duality to another physical $T$ interval, $1 \leq a_{d} \leq \infty$, of the same model on $\Lambda_{d}$.)

From the above observation we can derive several interesting results. First, from eq. (5), it follows that there is an exact 1-1 correspondence between the CT singularities of the $q \mathrm{PM}$ on $\Lambda_{d}$ in the interval $-\infty \leq a_{d} \leq-(q-1)$ and the physical singularities of the $q$-state Potts $\mathrm{AF}$ on $\Lambda$. In particular, if the $q \mathrm{PAF}$ on $\Lambda$ has a phase transition at $a_{P M-A F M}$ (here, $\mathrm{PM}=$ paramagnetic), then there is a corresponding CT singularity in $f\left(\Lambda_{d}, q, a\right)$ at $a=a_{\ell}$, where

$$
a_{\ell}=\mathcal{D}\left(a_{P M-A F M}\right)
$$

and this is the leftmost CT singularity in the interval $-\infty<a_{d} \leq 0$ (hence the label $\ell$ ). These transitions are of the same order. If the PM-AFM transition of the $q \mathrm{PAF}$ on $\Lambda$ is continuous, then the singularity in $f$ of the $q \mathrm{PM}$ at the CT point $a_{\ell}$ is the same when approached from the left or from the right and, writing $f\left(\Lambda_{d}, q, a\right)_{\text {sing }} \sim\left|a-a_{\ell}\right|^{2-\alpha_{\ell}}$, we have $\alpha_{\ell}=\alpha=\alpha^{\prime}$, where the exponent $\alpha=\alpha^{\prime}$ is the physical specific heat exponent of the $q$-state PAF at 
the PM-AFM transition. (In general we cannot use duality to relate the $\beta$ or $\gamma$ exponents for the staggered magnetization and susceptibility of the $q$-state PAF at $a_{P M-A F M}$ on $\Lambda$ and staggered or uniform $\beta$ or $\gamma$ exponents at $a_{\ell}$ since the duality applies in the absence of external field(s).) Now for a model above its lower critical dimensionality, so that there is a symmetry-breaking phase transition, a general property of the CT phase diagram is that in the complex $a$ plane, the continuous locus of points $\mathcal{B}$ where the free energy is nonanalytic (which determines the CT phase boundaries) is compact, i.e., the region sufficiently far from the origin is in the complex-temperature extension of the FM phase [5, 7]. (Henceforth, we shall use (CTE)FM to denote the CT extension of the FM phase, and so forth for other phases.) It follows that if the $q \mathrm{PAF}$ on $\Lambda$ has a PM-AFM transition, then since $a_{\ell}$ is the leftmost singularity in the $a_{d}$ plane of the $q$-state Potts model on $\Lambda_{d}$, the CT phase to the left of $a_{\ell}$ is the (CTE)FM phase of the latter model.

It may be helpful to illustrate these general results briefly for the known $q=2$ case. For the square lattice, $\Lambda=\Lambda_{d}$ and $a_{s q, q=2, P M-A F M}=\sqrt{2}-1$, so that $a_{s q, q=2, \ell}=-(\sqrt{2}+1)$. In the terminology of Ref. [7], the CT phase to the right of $a_{s q, q=2, \ell}$ is an O phase, meaning that it is not the CT extension of any physical phase. For the honeycomb (hc) lattice, $a_{h c, q=2, P M-A F M}=2-\sqrt{3}$, so that the leftmost CT singularity of the Ising model on the dual, triangular ( $\mathrm{t}$ ) lattice is at $a_{t, q=2, \ell}=-\sqrt{3}$. For the Ising model on the honeycomb lattice, the phases on the left and right of $a_{h c, q=2, \ell}$ are the (CTE)FM and (CTE)AFM phases; for the Ising model on the triangular lattice, the phases to the left and right of $a_{t, q=2, \ell}$ are the (CTE)FM and an O phase, respectively [14. The analyticity of $f$ on the he lattice in the interval $-\infty<a_{d}<-1$ is now seen as being equivalent to the absence of a finite- $T$ transition in the Ising $\mathrm{AF}$ on the triangular lattice. On the kagomé lattice, $a_{k a g, q=2, \ell}=$ $-3^{1 / 4}(2-\sqrt{3})^{-1 / 2}$ [15, 16], separating the (CTE)FM and (CTE)PM phases on the left and right, and corresponding to the PM-AFM transition of the Ising AF on the dual diced lattice.

A second type of behavior occurs if the $q \mathrm{PAF}$ on $\Lambda$ has no finite- $T$ phase transition but is critical at $T=0$; this is associated with a part of the CT phase boundary $\mathcal{B}$ passing through $a=0$ in the CT phase diagram of this model. It then follows that for the $q$-state Potts model on $\Lambda_{d}$ a part of the respective $\mathcal{B}$ for that model passes through $a_{d}=-(q-1)$. As examples, the Ising AF on the triangular lattice, and the $q=3$ Potts AF on the square and kagomé lattices all have zero-temperature critical points, so that there are respective singular points

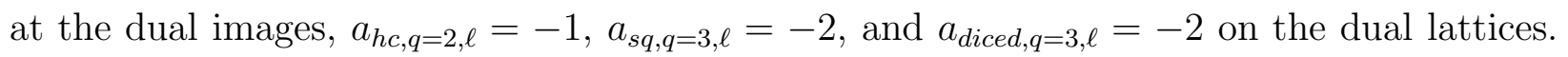
As discussed for the square lattice in Ref. [17], $a_{s q, q=3, \ell}=-2$ is the leftmost point where the $\mathrm{CT}$ phase boundary $\mathcal{B}$ crosses the negative real axis (see also Refs. [10, 18]).

As the third type of behavior, if the $q$-state Potts AF on $\Lambda$ has no finite-temperature phase transition and is also not critical at $T=0$ (which is manifested by $\mathcal{B}$ not passing 
through $a=0$ in the complex $a$ plane), then it follows that $f\left(\Lambda_{d}, q, a_{d}\right)$ is analytic in the CT interval $-\infty<a_{d} \leq-(q-1)$, which therefore must be part of the (CTE)FM phase of the $q \mathrm{PM}$ on $\Lambda_{d}$. This type of behavior occurs, for example, for the Potts model on the square lattice with $q \geq 4$.

We consider now the $q=3$ Potts model on the honeycomb and triangular lattices. Usually, in cases where there are no exact solutions, the locations of CT singular points are most accurately determined from series analyses; indeed, the complications due to these singularities were recognized in early work on series [4]. However, for $a_{\ell}$, one has a powerful alternative approach: to locate it using eq. (6) and a precise determination of the physical PM-AFM transition of the $q \mathrm{PAF}$ on the dual lattice. This is our first application. For the $q=3 \mathrm{PAF}$ on the triangular lattice, Monte Carlo and series analyses [19]-[21] led to the conclusion that this model has a weakly first order transition. The most recent study [21], using Monte Carlo methods, has yielded a very precise determination of the transition point: $T_{t}=0.62731 \pm 0.00006$, i.e., $a_{P M-A F M, t}=0.20309 \pm 0.00003$. We infer that the $q=3$ Potts model on the honeycomb lattice has a corresponding singularity at the CT point

$$
a_{h c, q=3, \ell}=\mathcal{D}\left(a_{P M-A F M, t}\right)=-(2.76454 \pm 0.00015)
$$

i.e., $z_{h c, q=3, \ell}=a_{h c, q=3, \ell}^{-1}=-(0.36172 \pm 0.00002)$. This value is in agreement with, and is more accurate than, the value $z_{h c, q=3, \ell}=-(0.363 \pm 0.003)$ obtained from a recent low-temperature series analysis in Ref. [22] (where $z_{h c, q=3, \ell}$ is denoted $u_{-}$). If one accepts the reported result [19]-21] that the PM-AFM transition of the $q=3$ PAF on the triangular lattice is (weakly) first order, the same applies for the singularity at $a_{h c, q=3, \ell}$, and, furthermore, these transitions have the same value of the latent heat. If one were formally to assign an exponent $\alpha$ to these transitions, it would thus be $\alpha=1$. Past experience shows that it can be difficult to distinguish a weakly first order transition from a continuous one, and indeed the series analysis in Ref. [22] found evidence for a continuous transition at $z_{h c, q=3, \ell}$ with $\alpha \simeq 0.5$.

A second application is to test a recent conjecture for an exact analytic value of the CT singularity at $z_{h c, q=3, \ell}$ made in Ref. [23], viz., that $z_{h c, q=3, \ell}=\cos (2 \pi / 9)-3^{1 / 2} \sin (2 \pi / 9)=$ $-.347296355 .$. , i.e., $a_{h c, q=3, \ell}=-2.87938524 \ldots$ (arising as a root of the equation $z^{3}-3 z-1=$ 0 ). Since the result of Ref. [22 was $5 \sigma$ (where $\sigma=0.003$ was the uncertainty in the location of the singular point) away from this conjecture, it was concluded that this conjecture is unlikely but not impossible to be correct. We can strengthen this conclusion here: our determination of $z_{h c, q=3, \ell}$ decisively refutes the conjecture of Ref. [23].

As a third application, we use our recent study of the $q=3$ Potts AF on the honeycomb lattice [24], where we found that this model has no finite-temperature PM-AFM transition and is, indeed, disordered at $T=0$, with nonzero ground state entropy $S_{0} / k_{B}=0.5068(3)$. 
From our discussion above, this implies that the free energy of the $q=3$ Potts model on the triangular lattice is analytic in the interval $-\infty<a \leq-2$. In passing, we note that this rules out yet another conjecture [25], that there could be a singularity in this model at the largest negative root of the equation $a^{3}+6 a^{2}+3 a-1=0$, viz., $a=-5.411474 \ldots$ (associated with the completion of complex-conjugate branches of CT zeros labelled 6 in Ref. 25]). Our result also implies that for the $q=3$ Potts model on the triangular lattice the leftmost component of the $\mathrm{CT}$ phase boundary $\mathcal{B}$ that crosses the real $a$ axis must do so at $a>-2$. These results apply a fortiori to the case $q \geq 4$ : in this range, the Potts $\mathrm{AF}$ on the honeycomb lattice has no finite- $T$ phase transition and is disordered, with finite entropy, at $T=0$, so that for each respective value of $q$, the corresponding Potts model on the triangular lattice is analytic in the range $-\infty<a \leq-(q-1)$. These results are borne out by explicit calculations of CT zeros of the partition function for $q$-state Potts models on honeycomb and kagomé lattices, to be presented elsewhere.

A fourth application concerns the $q$-state PAF on the diced lattice (dual of kagomé). Ref. 22. found CT singularities at $z_{k a g, q=3, \ell}=-0.4023 \pm 0.0005$ and $z_{k a g, q=4, \ell}=-0.42 \pm 0.01$ for the $q=3$ and $q=4$ Potts model on the kagomé lattice. From the first of these, we calculate that the $q=3$ PAF on the diced lattice has a PM-AFM phase transition at

$$
a_{\text {diced }, q=3, P M-A F M}=0.1393 \pm 0.0008
$$

For comparison, from the exact value for $a_{k a g, q=2, \ell}$, we have $a_{\text {diced }, q=2, P M-A F M}=0.4354205 \ldots$ for the Ising case. Furthermore, we find the interesting result that the $q=4$ Potts AF on the diced lattice has no finite- $T$ phase transition and also is not critical at $T=0$, since

$$
\mathcal{D}\left(a_{k a g, q=4, \ell}\right)=-(0.18 \pm 0.02)
$$

is negative. Remarkably, we have thus used information on a CT singular point of the Potts model on one lattice to derive a new physical result for this model on the dual lattice. From this we also determine the critical integer value of $q$ for the $q$-state Potts antiferromagnet on the diced lattice above which it has no finite-temperature phase transition, viz., $q=3$.

In summary, we have used an exact duality property of a $2 \mathrm{D} q$-state Potts model to relate certain complex-temperature properties of the model on a given lattice to physical properties of the Potts antiferromagnet on the dual lattice. This result gives useful information about the complex-temperature phase diagrams of $2 \mathrm{D}$ Potts models, and we have been able to use it to rule out two conjectures and to find the critical integer value of $q$ for the Potts antiferromagnet on the diced lattice.

This research was supported in part by the NSF grant PHY-93-09888. R.S. thanks Prof. Tony Guttmann for giving us a copy of Ref. [22] prior to publication. 


\section{References}

[1] Yang, C. N. and Lee, T. D. 1952 Phys. Rev. 87 404; Lee, T. D. and Yang, C. N. 1952 ibid 87410 .

[2] Fisher, M. E. 1965 Lectures in Theoretical Physics (Univ. of Colorado Press), vol. 7C, p. 1.

[3] Katsura, S. 1967 Prog. Theor. Phys. 38, 1415; Abe, R. 1967 Prog. Theor. Phys. 38, 322; Ono, S., Karaki, Y., Suzuki, M., and Kawabata, C. 1968 J. Phys. Soc. Jpn. 25, 54.

[4] Domb, C. and Guttmann, A. J. 1970 J. Phys. C 31652.

[5] Matveev, V. and Shrock, R. 1995 J. Phys. A 28 4859; Matveev, V. and Shrock, R. 1996 Phys. Rev. E53, 254; 1996 Phys. Lett. A215 271.

[6] Marchesini, G. and Shrock, R. 1989. Nucl. Phys. B318 541.

[7] Matveev, V. and Shrock, R. 1995 J. Phys. A 281557.

[8] Potts, R. B. 1952 Proc. Camb. Phil. Soc. 48106.

[9] Wu, F. Y. 1982 Rev. Mod. Phys. 54235.

[10] Martin, P. P. 1991 Potts Models and Related Problems in Statistical Mechanics (World Scientific, Singapore).

[11] Itzykson, C., Saleur, H., and Zuber, J.-B., eds. 1988 Conformal Invariance and Applications to Statistical Mechanics (World Scientific, Singapore).

[12] Onsager, L. 1944 Phys. Rev. 65 117; Yang, C. N. 1952 Phys. Rev. 85808.

[13] Kim, D. and Joseph, R. J. 1974 J. Phys. C 7, L167; Burkhardt, T. W. and Southern, B. W. 1978 J. Phys. A 11 L247.

[14] Matveev, V. and Shrock, R. 1996 J. Phys. A 29803.

[15] Abe, R., Dotera, T., and Ogawa, T. 1991 Prog. Theor. Phys. 85509.

[16] Matveev, V. and Shrock, R. 1995 J. Phys. A 285235.

[17] Matveev, V. and Shrock, R. 1996 Phys. Rev. E54 6174.

[18] Chen, C. N., Hu, C. K, and Wu, F. Y. 1996 Phys. Rev. Lett. 76169. 
[19] Grest, G. S. 1981 J. Phys. A 14 L217; Saito, Y. 1982 J. Phys. A 151885.

[20] Enting, I. G. and Wu, F. Y. 1982 J. Stat. Phys. 28351.

[21] Adler, J., Brandt, A., Janke, W., and Shmulyian, S. 1995 J. Phys. A 28, 5117.

[22] Jensen, I., Guttmann, A. J., and Enting, I. G. 1997 "The Potts Model on Kagomé and Honeycomb Lattices", J. Phys. A, to appear.

[23] Maillard, J.-M. Computers and Mathematics with Applications, to appear.

[24] Shrock, R. and Tsai, S.-H. 1997 J. Phys. A 30 495; Phys. Rev. E55 5165; ibid 6791.

[25] Martin, P. P. and Maillard, J.-M. 1986 J. Phys. A 19 L547. 